\title{
Etude chimique du smen marocain
}

\author{
A. EL MARRAKCHI*, M. BERRADA**, M. CHAHBOUN** \\ et M. BENBOUHOU* \\ - Département d'Hygiène et Industrie des Denrées alimentaires \\ d'Origine animale (HIDAOA), Institut Agronomique et Vétérinaire Hassan II, \\ B.P. 6 202-Rabat-Instituts, Maroc \\ ** Section de Technologie alimentaire du même Institut
}

\begin{abstract}
Résumé
L'étude chimique du smen montre que la composition en acides gras totaux et en insaponifiable reste la même que celle du beurre fermier. Cependant, ce produit connaît une forte hydrolyse. Cette lipolyse est sélective : les acides gras à courte chaîne sont libérés préférentiellement, en particulier l'acide butyrique, suivi des acides gras insaturés avec une attaque plus rapide de l'acide oléique. Les acides gras saturés sont libérés en dernier. Au sein de cette fraction, les acides myristique et palmitique dominent.

Malgré le salage et l'acidité, deux facteurs favorisant l'oxydation, le smen reste peu oxydé. Ceci peut s'expliquer par le mode de conditionnement du produit pendant sa conservation.

Les composants de la phase aqueuse subissent, d'une façon générale, une diminution qui est le résultat, soit d'une dégradation microbienne, soit d'une perte avec de l'eau exsudée.

Le diacétyle, substance responsable de l'arôme du beurre, est détruit au cours de l'élaboration du produit ; il n'est donc pas question de lui attribuer un rôle quelconque dans l'explication de la flaveur du smen.
\end{abstract}

Mots clés : Smen - Lipolyse - Evolution au cours de la conservation.

\section{Summary}

\section{Chemical study of moroccan smen}

The chemical investigations on "smen " showed that total fatty acids and unsaponifiable fractions are similar to the corresponding fractions in butter. Although smen is relatively a hydrolyzed product, the lipolysis taking place is a selective process. The short chain fatty acids, particularly butyric acid, are preferentially liberated. The insaturated fatty acids are liberated to an intermediate extent with a more rapid rate on oleic acid. The saturated fatty acids are liberated at the final steps of lipolysis. Within this fraction, myristic and palmitic acids predominate.

Despite salting and development of acidity, both favoring oxydation, smen is relatively non oxydized product. This might be explained by the way it is stored.

The aqueous phase components are generally reduced. This reduction results either from a microbial degradation or a loss in the exsudate.

Diacetyl, which is responsible for the flavor of butter, is destroyed during the elaboration of smen. Therefore, Diacetyl doesn't contribute to the flavor of smen.

Key words : Smen - Lipolysis - Composition - Evolution during storage. 
Le terme «smen » désigne, généralement, des produits préparés, de façon artisanale, à partir du beurre fermier par lavage et salage, puis conditionnement dans des pots en terre et conservation à l'abri de l'air et de la lumière pour une durée variable d'au moins 6 mois. Cette préparation fait ressortir les caractéristiques suivantes : absence de tout traitement thermique, le salage constituant le seul élément de conservation. Les conditions de stockage sont également originales (anaérobiose et température ambiante).

Cette technologie générale, pratiquée dans tout le Maroc, est susceptible de quelques modifications selon les provinces :

- le beurre fermier peut être fabriqué à partir d'un mélange de lait de vache et de chèvre. C'est ce mode de préparation qui est effectué dans les régions du Nord et du Sud du Maroc, là où l'élevage caprin prédomine ;

- avant le salage, le beurre peut exceptionnellement subir une cuisson de $1 \mathrm{~h}$ à $1 \mathrm{~h} 30$ pour favoriser une certaine évaporation de l'eau. Ce type de smen est uniquement fabriqué à Marrakech ;

- le smen, après salage mais avant son conditionnement, peut être aromatisé de thym sous forme de fragments de la plante ou d'extrait.

Ce produit est très apprécié pour ses qualités gustatives, diététiques, voire thérapeutiques, mais la production nationale est insuffisante pour satisfaire la demande sans cesse croissante. Pour combler ce déficit partiellement résolu par l'importation de beurre salé abusivement dénommé smen, il est nécessaire de promouvoir cette production, ce qui nécessite la connaissance précise de ce produit sur le plan de sa composition chimique. D'autre part, la qualité nutritionnelle de cet aliment est méconnue ce qui justifie davantage le présent travail qui porte sur l'étude du smen de commerce ainsi que sur le produit au cours de son élaboration.

\section{Matériel et méthodes}

Les méthodes utilisées ont pour objet, d'une part, de mettre en évidence et de doser les divers constituants du smen, d'autre part, de montrer les modifications éventuelles subies par ce produit, au cours de sa préparation et de sa conservation, par divers processus tels que l'oxydation, l'hydrolyse et la formation de substances volatiles.

\section{A. Composition globale}

1. Composition de la phase grasse

a) Etude de la phase saponifiable : composition en acides gras totaux (A.G.T.)

La préparation de l'échantillon et l'analyse sont conduites selon la méthode de Wolf (1968). Les conditions de la chromatographie des esters méthyliques ont été les suivantes :

- appareil : Hewlett Packard 5730 A ; 
- détecteur à ionisation de flamme ;

- colonne en acier inoxydable, $1,80 \mathrm{~m}$ de long et $3 \mathrm{~mm}$ de diamètre ;

- support Chromosorb W.A.W. 80-100 mesh ;

- phase stationnaire : polysuccinate de diéthylène glycol à $10 \%$ sur le Chromosorb ;

- température du four : programmée de $60^{\circ} \mathrm{C}$ à $180^{\circ} \mathrm{C}$ à raison de $4^{\circ} \mathrm{C}$ par minute ;

- température de l'injecteur et du détecteur : $250^{\circ} \mathrm{C}$;

- débit du gaz vecteur (azote) : $30 \mathrm{ml}$ par minute ;

- quantité injectée : $0,2 \mathrm{ml}$;

- enregistreur : déroulement du papier $0,3 \mathrm{~cm}$ par minute.

L'identification des pics des chromatogrammes des esters méthyliques d'acides gras de nos échantillons a été faite par comparaison de leur temps de rétention avec celui d'esters d'acides gras standards.

Les pourcentages en masse ont été calculés par la mesure des surfaces respectives des pics. Chaque surface a été mesurée par triangulation à partir du produit de la hauteur par la largeur à la mi-hauteur du pic.

b) Etude de la phase grasse insaponifiable

I

- Détermination de l'insaponifiable total

Pour la saponification de la matière grasse, nous avons suivi la technique décrite par WoLF (1968).

- Dosage des stérols

Il a été réalisé par précipitation à la digitonine selon la norme de la Fédération Internationale de Laiterie (F.I.L.-I.D.F. 32, 1965).

2. Composition de la phase non grasse

a) Teneur en eau

Le dosage de l'eau est fait par entraînement azéotropique selon le procédé AFNOR (N.F. T 60-201).

b) Teneur en extrait sec dégraissé (E.S.D.).

Après fusion du smen, l'extraction de la matière grasse a été réalisée par l'éther de pétrole et le résidu de filtration est séché à l'étuve réglée à $150^{\circ} \mathrm{C}$ jusqu'à poids constant.

c) Teneur en chlorures

La méthode utilisée est celle de Charpentier-Volhard.

d) Teneur en lactose

La teneur en lactose de la phase non grasse est déterminée selon la méthode de BERTRAND.

e) Teneur en protéines

Nous avons appliqué la méthode de KJELDAHL. 


\section{B. Mesure du degré d'hydrolyse}

L'appréciation du degré de lipolyse est réalisée globalement par titrimétrie et qualitativement par la détermination de la composition en acides gras libres (A.G.L.).)

\section{Mesure de 'indice d'acide}

Le dosage des A.G.L. est fait en milieu éthanolique par la potasse selon la technique de Wolf (1968).

\section{Composition en A.G.L.}

La détermination de la composition en A.G.L. est réalisée en chromatographie en phase gazeuse, décrite précédemment, après extraction des A.G.L. par la méthode de KuzDzaL et al. (1971).

\section{Mesure de l'oxydation}

L'importance de l'oxydation est évaluée par la mesure de l'indice de peroxyde et par la composition en carbonylés totaux.

Nous avons suivi encore, dans ces deux cas, les techniques décrites par WoLF (1968).

\section{Analyse de quelques substances volatiles}

Nous rangeons dans cette rubrique, les dosages de l'acétoïne et du diacétyle par colorimétrie. La méthode utilisée est celle de PrILl et HAMMER (1968).

\section{Résultats}

\section{A. Etude du smen de commerce}

Dix-huit échantillons de smen ont été analysés du point de vue chimique. A titre comparatif, nous avons analysé un échantillon de beurre salé d'importation (vendu sous l'appellation de smen) et un échantillon de beurre fermier.

\section{Composition chimique globale}

Le tableau 1 donne la composition chimique globale du smen. Les valeurs présentées sont exprimées en pour cent du produit pour les teneurs en matière grasse, en extrait sec dégraissé, en eau, en stérols et en sel, en pour cent de l'insaponifiable pour les stérols et en pour cent de la fraction aqueuse pour le sel, le lactose et les protéines.

A l'analyse du tableau 1 on constate que :

- la teneur moyenne en eau est de $13,7 \%$ du produit mais varie dans des limites assez larges (de 10 à $20 \%$ ). Les teneurs rencontrées, comparées 
à celles du beurre, matière première du smen, montrent qu'elles sont toujours plus faibles ;

- la teneur en extrait sec dégraissé est en moyenne de 4,96\% avec des valeurs extrêmes de 2,73 à 7,36 ;

- la teneur en matière grasse est en moyenne de $81,34 \%$ et varie d'une manière inverse à celle des teneurs en eau ;

- la teneur en sel $(\mathrm{NaCl})$ est en moyenne de 1,5\% avec des valeurs extrêmes de 0,9 à $3,4 \%$. Rapportée à la phase aqueuse, cette teneur s'élève à $8 \%$ en moyenne, variant de 3,4 à $19 \%$;

- les teneurs en lactose et en protéines sont inférieures à celles du beurre et représentent respectivement 1,22 et $3,25 \%$ du non gras ;

- les teneurs en insaponifiable et en stérols sont respectivement de 0,33 et $0,15 \%$ de la matière grasse totale et sont comparables à celles du beurre fermier.

\section{Composition en acides gras totaux (A.G.T.)}

Les résultats d'analyse chromatographique des échantillons de smen sont consignés dans le tableau 2 . Les pourcentages relatifs aux acides $\mathrm{C}_{18}$ : 3 et $\mathrm{C}_{20}$ : 0 sont présentés ensemble, parce que dans nos conditions expérimentales, la séparation de ces acides n'a pu être possible. Cette difficulté proviendrait du fait que, comme le souligne Wolf (1968), sur le succinate de diéthylène glycol, l'ordre d'élution des esters linolénique et arachidique est variable avec la température et le taux d'imprégnation de la colonne.

La composition en A.G.T. est, presque, la même pour tous les échantillons. En moyenne, les acides gras saturés représentent $66,74 \%$ des acides totaux, les acides gras mono-insaturés $31,18 \%$ et les acides gras di-insaturés $2,92 \%$. Comparée à celle du beurre salé et du beurre fermier, la composition moyenne en A.G.T. du smen est presque identique.

\section{Degré d'hydrolyse}

L'indice d'acide moyen exprimé en milligramme de potasse par gramme de matière grasse est de 52,3. Il montre que tous les produits analysés accusent une forte lipolyse. Les valeurs extrêmes enregistrées varient de 12,1 à 107,7 (tabl. 1). Cependant, l'analyse chromatographique des acides gras libres (A.G.L.) montre que l'hydrolyse est pratiquement identique pour tous les échantillons. Ceci suggère que la lipolyse observée est sélective. Par rapport à la composition en A.G.T., la teneur en acides gras à courte chaîne de $\mathrm{C}_{4}$ à $\mathrm{C}_{10}$ est diminuée, celle des acides gras saturés de $\mathrm{C}_{12}$ à $\mathrm{C}_{18}$ également diminuée, enfin la teneur des acides gras insaturés est augmentée. Parmi les acides gras à courte chaîne, ce sont essentiellement les acides butyrique et caproïque qui manifestent une nette diminution. La teneur en acide caprique est augmentée, alors que celle de l'acide caprylique reste inchangée (tab. 2).

\section{Degré d'oxydation}

Les résultats du tableau 1 montrent que, d'une façon globale, le smen est peu oxydé. L'indice moyen de peroxyde est de $3,6 \mathrm{meq} / \mathrm{kg}$ avec des valeurs 
TABleau 1 - TABle 1

Composition et caractéristiques physico-chimiques du smen - Composition and physico-chemical characteristics of smen

\begin{tabular}{|c|c|c|c|c|}
\hline & \multicolumn{2}{|c|}{ Smen } & \multirow{2}{*}{$\begin{array}{l}\text { Beurre salé } \\
\text { importé }\end{array}$} & \multirow{2}{*}{$\begin{array}{c}\text { Beurre fermier } \\
\text { frais }\end{array}$} \\
\hline & $\mathrm{X}$ & Valeurs extrêmes & & \\
\hline Matière grasse totale ${ }^{+}$ & 81.34 & 76.39- 87.30 & 83.07 & 73.69 \\
\hline $\mathrm{Eau}^{+}$ & 13.7 & $10-20$ & 11.6 & 23.3 \\
\hline Extrait sec non gras ${ }^{+}$ & 4.96 & 2.73- 7.36 & 5.33 & 3.01 \\
\hline Chlorure de sodium ${ }^{+}$ & 1.5 & $0.9-3.4$ & 3.6 & 0.01 \\
\hline Chlorure de sodium ++ & 8.0 & $3.4-19.3$ & 20.3 & 0.036 \\
\hline Lactose $^{++}$ & 1.22 & $0.56-1.98$ & 3.46 & 3.48 \\
\hline Protéines ${ }^{++}$ & 3.25 & $2.23-4.02$ & 3.24 & 5.60 \\
\hline Insaponifiable +++ & 0.33 & $0.23-\quad 0.50$ & 0.33 & 0.30 \\
\hline $\begin{array}{l}\text { Stérols : } \\
\text { - en } \% \text { de l'insaponifiable } \\
\text { - en } \% \text { de la matière grasse totale }\end{array}$ & $\begin{array}{r}47.03 \\
0.15\end{array}$ & $\begin{array}{rr}35.88-57.16 \\
0.11-\quad 0.20\end{array}$ & $\begin{array}{r}42.43 \\
0.14\end{array}$ & $\begin{array}{r}42.22 \\
0.12\end{array}$ \\
\hline Indice d'acide & 52.34 & $12.14-107.79$ & 11.78 & 2.37 \\
\hline Indice de peroxyde (meq/kg) & 3.67 & 0.43- 24.95 & 1.35 & 0.53 \\
\hline \multicolumn{2}{|l|}{$\begin{aligned} \mathrm{X} & : \text { average. } \\
+ & : \text { expressed as percent of smen. } \\
++ & : \text { expressed as percent of non fat. } \\
+++ & : \text { expressed as percent of total fat }\end{aligned}$} & $\begin{aligned} \mathrm{X} & : \text { moyenne. } \\
+: & \text { exprimé en } \\
++ & : \text { exprimé en } \\
+++: & \text { exprimé en }\end{aligned}$ & $\begin{array}{l}\text { du smen. } \\
\text { du non gras } \\
\text { le la matière }\end{array}$ & totale (M.G.T.). \\
\hline
\end{tabular}


TABleau 2 - TABle 2

Composition en acides gras du smen (exprimée en \% en poids des acides totaux)

Fatty acid content of smen (expressed as weight percent of total fatty acids)

\begin{tabular}{|c|c|c|c|c|c|c|c|c|}
\hline & \multicolumn{4}{|c|}{ Acides gras totaux } & \multicolumn{4}{|c|}{ Acides gras libres } \\
\hline & \multicolumn{2}{|c|}{ Smen } & \multirow{2}{*}{$\begin{array}{l}\text { Beurre } \\
\text { salé }\end{array}$} & \multirow{2}{*}{$\begin{array}{l}\text { Beurre } \\
\text { fermier } \\
\text { frais }\end{array}$} & \multicolumn{2}{|c|}{ Smen } & \multirow{2}{*}{$\begin{array}{l}\text { Beurre } \\
\text { salé }\end{array}$} & \multirow{2}{*}{$\begin{array}{l}\text { Beurre } \\
\text { fermier } \\
\text { frais }\end{array}$} \\
\hline & $\mathrm{X}$ & $\begin{array}{l}\text { Valeurs } \\
\text { extrêmes }\end{array}$ & & & $\mathrm{X}$ & $\begin{array}{l}\text { Valeurs } \\
\text { extrêmes }\end{array}$ & & \\
\hline $\begin{array}{l}\mathrm{C}_{4}: 0 \\
\mathrm{C}_{6}: 0 \\
\mathrm{C}_{8}: 0 \\
\mathrm{C}_{10}: 0 \\
\mathrm{C}_{10}: 1 \\
\mathrm{C}_{12}: 0 \\
\mathrm{C}_{14}: 0 \\
\mathrm{C}_{14}: 1 \\
\mathrm{C}_{15}: 0 \\
\mathrm{C}_{16}: 0 \\
\mathrm{C}_{16}: 1 \\
\mathrm{C}_{18}: 0 \\
\mathrm{C}_{18}: 1 \\
\mathrm{C}_{18}: 2 \\
\mathrm{C}_{20}: 0+\mathrm{C}_{18}: 3\end{array}$ & $\begin{array}{r}2.43 \\
175 \\
1.16 \\
2.82 \\
0.21 \\
3.27 \\
10.95 \\
1.38 \\
1.20 \\
28.40 \\
1.82 \\
12.90 \\
27.77 \\
2.92 \\
1.86\end{array}$ & $\begin{array}{r}0.8-3.7 \\
0.8-2.2 \\
0.6-1.8 \\
2.4-3.8 \\
0.1-0.4 \\
2.9-4.6 \\
7.9-14.1 \\
0.6-2.6 \\
0.8-1.8 \\
24.5-30.3 \\
0.9-2.4 \\
8.7-15.9 \\
21.9-32.2 \\
0.9-6.2 \\
0.7-3.6\end{array}$ & $\begin{array}{r}2.9 \\
1.9 \\
1.5 \\
2.8 \\
0.3 \\
4.0 \\
13.3 \\
2.2 \\
1.3 \\
26.5 \\
0.8 \\
13.5 \\
25.1 \\
1.4 \\
2.1\end{array}$ & $\begin{array}{r}2.9 \\
1.9 \\
1.5 \\
2.7 \\
0.2 \\
2.9 \\
11.5 \\
1.6 \\
1.4 \\
30.7 \\
2.2 \\
14.7 \\
22.5 \\
2.2 \\
1.3\end{array}$ & $\begin{array}{r}0.45 \\
0.85 \\
1.15 \\
3.47 \\
0.30 \\
3.60 \\
10.5 \\
1.88 \\
1.27 \\
25.89 \\
4.40 \\
11.95 \\
29.03 \\
2.90 \\
2.41\end{array}$ & $\begin{array}{r}0.2-1.6 \\
0.2-5.0 \\
0.2-5.0 \\
1.6-9.0 \\
\text { T- } 0.9 \\
1.8-8.7 \\
5.3-16.6 \\
0.4-4.8 \\
0.6-2.7 \\
16.6-32.4 \\
1.7-14.5 \\
3.8-24.5 \\
16.3-43.1 \\
\text { T- } 5.5 \\
\text { T- } 4.1\end{array}$ & $\begin{array}{r}0.3 \\
0.3 \\
0.5 \\
1.8 \\
0.2 \\
3.3 \\
10.8 \\
1.6 \\
1.4 \\
30.1 \\
2.6 \\
14.8 \\
28.1 \\
2.2 \\
1.7\end{array}$ & $\begin{array}{r}0.3 \\
0.4 \\
0.7 \\
2.5 \\
0.2 \\
2.7 \\
9.8 \\
1.6 \\
0.9 \\
19.6 \\
2.0 \\
18.1 \\
31.9 \\
4.2 \\
3.7\end{array}$ \\
\hline $\begin{array}{l}\mathrm{X} \text { : moyenne. } \\
\mathrm{T} \text { : traces. }\end{array}$ & & & & $\begin{array}{l}\mathrm{X}: \\
\mathrm{T}:\end{array}$ & & & & \\
\hline
\end{tabular}


extrêmes de 0,43 à 24,95 . Bien que les variations soient de forte amplitude, $73 \%$ des échantillons ont un indice de peroxyde inférieur au chiffre moyen.

\section{B. Etude du smen au cours de son élaboration}

Cette étude a été réalisée sur deux échantillons du smen A et B, préparés au département d'Hidaoa de l'Institut Agronomique et Vétérinaire Hassan II à Rabat.

Le beurre, matière première du smen, était préparé à partir du lait cru produit à la ferme expérimentale de l'Institut.

Le lait était transporté dans des bidons préalablement lavés, désinfectés et rincés, puis transvasé à son arrivée dans des récipients propres pour y subir une coagulation. Celle-ci était, généralement, obtenue, à la température ambiante, après $48 \mathrm{~h}$.

Après coagulation, le barattage était effectué dans une jarre en terre cuite. Le beurre recueilli est ensuite lavé et malaxé.

Il est alors divisé en deux parties égales qui sont données à deux personnes pour la fabrication du smen.

La préparation du smen a nécessité trois étapes : un lavage, un salage et un conditionnement.

Le lavage a pour but de débarrasser le beurre de tout son Iben résiduel. Il est pratiqué à l'eau tiède puis à l'eau légèrement salée.

Après cette opération, le beurre lavé est salé avec du sel de cuisine. Le salage s'effectue en ajoutant progressivement le chlorure de sodium au beurre, en malaxant celui-ci à la main.

Après cette adjonction, le produit est mis dans des pots en terre qui sont fermés et entreposés dans un local à l'abri de l'air et de la lumière.

Des prélèvements sont effectués à $J_{0}$ (beurre avant salage), $J_{7}$ ( $7^{e}$ jour après salage), $J_{47}, J_{77}$ et $J_{123}$ pour les analyses chimiques.

\section{Composition chimique du smen}

Le tableau 3 donne la composition chimique globale des échantillons A et $\mathrm{B}$ de smen en fonction du temps de conservation. Les valeurs présentées sont les moyennes de deux analyses et sont exprimées en pour cent produit et en pour cent du non gras pour les teneurs en eau, extrait sec dégraissé (E.S.D.), sel, protéines, lactose et insaponifiable ; en pour cent du produit pour les teneurs en matière grasse et en pour cent de la matière grasse et de l'insaponifiable pour les stérols.

Les résultats obtenus montrent que le smen, au cours de son élaboration, subit des transformations chimiques importantes.

Les teneurs en eau, ainsi que les constituants de la phase aqueuse, diminuent de $J_{0}$ à $J_{123}$ sauf pour le sel dont la teneur augmente à $J_{7}$ pour décroître par la suite. Les teneurs en eau passent de $22,5 \%$ et $20 \%$ respectivement pour les échantillons $\mathrm{A}$ et $\mathrm{B}$ à $16,2 \%$ et $13,7 \%$. Cette perte globale repré- 
TABLEAU 3 - TABLE 3

Composition chimique du smen au cours de son élaboration. - Chemical composition of smen during its making

\begin{tabular}{|c|c|c|c|c|c|c|c|c|c|c|c|c|c|c|c|c|}
\hline \multirow{2}{*}{$\begin{array}{c}\text { Prélève- } \\
\text { ment }\end{array}$} & \multirow{2}{*}{$\begin{array}{c}\text { Echan- } \\
\text { tillon }\end{array}$} & \multicolumn{2}{|c|}{$\begin{array}{c}\text { Teneur en } \\
\text { eau }\end{array}$} & \multicolumn{2}{|c|}{$\begin{array}{c}\text { Teneur en } \\
\text { E.S.D. }\end{array}$} & \multirow{2}{*}{$\begin{array}{c}\begin{array}{c}\text { Teneur } \\
\text { en } \\
\text { M.G. }\end{array} \\
\text { a }\end{array}$} & \multicolumn{2}{|c|}{$\begin{array}{c}\text { Teneur en } \\
\text { sel }\end{array}$} & \multicolumn{2}{|c|}{$\begin{array}{l}\text { Teneur en } \\
\text { protéines }\end{array}$} & \multicolumn{2}{|c|}{$\begin{array}{l}\text { Teneur en } \\
\text { lactose }\end{array}$} & \multicolumn{2}{|c|}{$\begin{array}{l}\text { Teneur en } \\
\text { insaponi- } \\
\text { fiable }\end{array}$} & \multicolumn{2}{|c|}{$\begin{array}{l}\text { Teneur en } \\
\text { stérols }\end{array}$} \\
\hline & & $\mathrm{a}^{*}$ & $\mathrm{~b}$ & $\mathrm{a}$ & $\mathrm{b}$ & & $\mathrm{a}$ & $\mathrm{b}$ & $\mathrm{a}$ & b & $\mathrm{a}$ & $\mathrm{b}$ & $\mathrm{a}$ & b & $c$ & $\mathrm{~d}$ \\
\hline $\begin{array}{l}\text { Beurre } \\
\mathrm{J}_{0}\end{array}$ & $\begin{array}{l}\mathrm{A} \\
\mathrm{B}\end{array}$ & $\begin{array}{l}22,5 \\
20,0\end{array}$ & $\begin{array}{l}83,9 \\
83,3\end{array}$ & $\begin{array}{l}4,31 \\
3,99\end{array}$ & $\begin{array}{l}16,07 \\
16,63\end{array}$ & $\begin{array}{l}73,19 \\
76,01\end{array}$ & $\begin{array}{l}0,02 \\
0,04\end{array}$ & $\begin{array}{l}0,10 \\
0,17\end{array}$ & $\begin{array}{l}1,46 \\
1,11\end{array}$ & $\begin{array}{l}5,45 \\
4,68\end{array}$ & $\begin{array}{l}1,05 \\
1,09\end{array}$ & $\begin{array}{l}3,93 \\
4,56\end{array}$ & $\begin{array}{l}0,21 \\
0,22\end{array}$ & $\begin{array}{l}0,30 \\
0,30\end{array}$ & $\begin{array}{l}0,18 \\
0,19\end{array}$ & $\begin{array}{l}61,0 \\
53,0\end{array}$ \\
\hline $\begin{array}{l}\text { Smen } \\
\mathrm{J}_{7}\end{array}$ & $\begin{array}{l}\mathrm{A} \\
\mathrm{B}\end{array}$ & $\begin{array}{l}20,0 \\
16,2\end{array}$ & $\begin{array}{l}78,3 \\
76,3\end{array}$ & $\begin{array}{l}5,32 \\
5,02\end{array}$ & $\begin{array}{l}21,63 \\
23,65\end{array}$ & $\begin{array}{l}74,68 \\
78,78\end{array}$ & $\begin{array}{l}1,02 \\
0,95\end{array}$ & $\begin{array}{l}4,03 \\
4,46\end{array}$ & $\begin{array}{l}1,11 \\
0,79\end{array}$ & $\begin{array}{l}4,37 \\
3,74\end{array}$ & $\begin{array}{l}0,70 \\
0,73\end{array}$ & $\begin{array}{l}2,75 \\
3,46\end{array}$ & $\begin{array}{l}0,23 \\
0,25\end{array}$ & $\begin{array}{l}0,31 \\
0,32\end{array}$ & $\begin{array}{l}0,18 \\
0,15\end{array}$ & $\begin{array}{l}59,6 \\
48,1\end{array}$ \\
\hline $\begin{array}{l}\text { Smen } \\
\mathrm{J}_{47}\end{array}$ & $\begin{array}{l}\mathrm{A} \\
\mathrm{B}\end{array}$ & $\begin{array}{l}17,5 \\
15,0\end{array}$ & $\begin{array}{l}75,8 \\
73,4\end{array}$ & $\begin{array}{l}5,56 \\
5,42\end{array}$ & $\begin{array}{l}24,11 \\
26,54\end{array}$ & $\begin{array}{l}76,94 \\
79,94\end{array}$ & $\begin{array}{l}0,86 \\
0,72\end{array}$ & $\begin{array}{l}3,73 \\
3,52\end{array}$ & $\begin{array}{l}0,42 \\
0,46\end{array}$ & $\begin{array}{l}1,86 \\
2,26\end{array}$ & $\begin{array}{l}0,61 \\
0,64\end{array}$ & $\begin{array}{l}2,65 \\
3,17\end{array}$ & $\begin{array}{l}0,24 \\
0,25\end{array}$ & $\begin{array}{l}0,32 \\
0,32\end{array}$ & $\begin{array}{l}0,20 \\
0,17\end{array}$ & $\begin{array}{l}62,5 \\
54,4\end{array}$ \\
\hline $\begin{array}{l}\text { Smen } \\
\mathrm{J}_{77}\end{array}$ & $\begin{array}{l}\mathrm{A} \\
\mathrm{B}\end{array}$ & $\begin{array}{l}16,0 \\
13,7\end{array}$ & $\begin{array}{l}73,2 \\
70,7\end{array}$ & $\begin{array}{l}5,87 \\
5,67\end{array}$ & $\begin{array}{l}26,84 \\
29,27\end{array}$ & $\begin{array}{l}78,13 \\
80,63\end{array}$ & $\begin{array}{l}0,72 \\
0,55\end{array}$ & $\begin{array}{l}3,29 \\
2,49\end{array}$ & $\begin{array}{l}0,13 \\
0,09\end{array}$ & $\begin{array}{l}0,62 \\
0,47\end{array}$ & $\begin{array}{l}0,31 \\
0,37\end{array}$ & $\begin{array}{l}1,42 \\
1,91\end{array}$ & $\begin{array}{l}0,26 \\
0,26\end{array}$ & $\begin{array}{l}0,34 \\
0,33\end{array}$ & $\begin{array}{l}0,22 \\
0,17\end{array}$ & $\begin{array}{l}64,7 \\
53,6\end{array}$ \\
\hline $\begin{array}{l}\text { Smen } \\
J_{123}\end{array}$ & $\begin{array}{l}\mathrm{A} \\
\mathrm{B}\end{array}$ & $\begin{array}{l}16,2 \\
13,7\end{array}$ & $\begin{array}{l}73,9 \\
70,2\end{array}$ & $\begin{array}{l}5,71 \\
5,84\end{array}$ & $\begin{array}{l}26,0 \\
29,81\end{array}$ & $\begin{array}{l}78,09 \\
80,46\end{array}$ & $\begin{array}{l}0,77 \\
0,66\end{array}$ & $\begin{array}{l}3,50 \\
3,37\end{array}$ & $\begin{array}{l}0,09 \\
0,07\end{array}$ & $\begin{array}{l}0,44 \\
0,38\end{array}$ & $\begin{array}{l}0,23 \\
0,27\end{array}$ & $\begin{array}{l}1,07 \\
0,91\end{array}$ & $\begin{array}{l}0,27 \\
0,26\end{array}$ & $\begin{array}{l}0,35 \\
0,33\end{array}$ & $\begin{array}{l}0,23 \\
0,18\end{array}$ & $\begin{array}{l}65,7 \\
54,5\end{array}$ \\
\hline \multicolumn{17}{|c|}{$\begin{array}{l}\text { *a : en } \% \text { du produit. } \\
\mathrm{b}: \text { en } \% \text { du non gras. } \\
\mathrm{c}: \text { en } \% \text { de la matière grasse. } \\
\mathrm{d}: \text { en } \% \text { de l'insaponifiable. }\end{array}$} \\
\hline
\end{tabular}


sente, en valeur relative, $6,9 \%$ pour les deux échantillons. Parallèlement, nous assistons à une augmentation de la teneur en matière grasse et en E.S.D. les teneurs en insaponifiable et en stérols restent pratiquement inchangées.

\section{Mesure du degré de lipolyse}

Les tableaux 4 et 5 donnent pour les deux échantillons A et B, l'indice exprimé en milligramme de potasse par gramme de matière grasse (M.G.) et la composition en A.G.T. et en A.G.L. exprimée en pour cent.

TABleaU 4 - TABLE 4

Détermination de l'indice d'acide (en mg potasse/g de M.G.)

Determination of acid index

\begin{tabular}{|c|c|c|}
\hline & Echantillon A & Echantillon B \\
\hline Beurre $\mathrm{J}_{0}$ & 1,4 & $0,70^{\circ}$ \\
\hline Smen $_{1} J_{7}$ & 3,56 & 1,43 \\
\hline $\operatorname{Smen}_{2} \quad J_{47}$ & 8,21 & 4,10 \\
\hline $\mathrm{Smen}_{3} \mathrm{~J}_{77}$ & 11,02 & 6,48 \\
\hline Smen $_{4} \quad J_{123}$ & 14,16 & 11,37 \\
\hline
\end{tabular}

Ces résultats montrent qu'au cours de la conservation, l'indice d'acide augmente de 1,4 à $\mathrm{J}_{0}$, pour l'échantillon $\mathrm{A}$ et 0,70 pour l'échantillon $\mathrm{B}$ à, respectivement, 14,16 et 11,37 à $\mathrm{J}_{123}$. Cette augmentation est le témoin d'une forte lipolyse confirmée par l'analyse chromatographique des acides gras libres.

Pour mieux suivre la cinétique de l'attaque des lipides, nous distinguerons les acides gras du smen en trois grands groupes : les acides gras à courte chaîne (A.G.C.) qui comprennent les acides butyrique, caproïque, caprylique et caprique ; les acides gras saturés à moyenne et longue chaîne (A.G.S.) qui sont représentés par les acides laurique, myristique, palmitique, stéarique et arachidique et enfin, les acides gras insaturés à moyenne et longue chaîne (A.G.I.) qui sont constitués par les acides myristoléique, oléique, linoléique et linolénique. Le tableau 5 rend compte de l'évolution de ces trois grands groupes d'acides gras.

Les A.G.C. représentent 10 à $11 \%$ des A.G.T. du beurre et $0 \%$ des A.G.L. à $\mathrm{J}_{0}$. $\mathrm{A} \mathrm{J}_{7}$, ils constituent toujours 10 à $11 \%$ des A.G.T. mais $18,24 \%$ et $10,79 \%$ des A.G.L. respectivement pour les échantillons A et B. Parmi les A.G.L., l'acide butyrique domine ; il représente $100 \%$ des A.G.C. de l'échantillon B et près de $64 \%$ des A.G.C. de l'échantillon A. Il est visible que les A.G.C. 
TABleau 5 - TABLE 5

Evolution des acides gras au cours de l'élaboration du smen (exprimée en poids des acides totaux)

Fatty acid evolution during making of smen (expression as weight percent of total acids)

\begin{tabular}{|c|c|c|c|c|c|c|c|c|c|c|c|}
\hline & & \multicolumn{2}{|c|}{$\mathrm{J}_{0}$} & \multicolumn{2}{|c|}{$\mathrm{J}_{7}$} & \multicolumn{2}{|c|}{$\mathrm{J}_{47}$} & \multicolumn{2}{|c|}{$\mathrm{J}_{77}$} & \multicolumn{2}{|c|}{$\mathrm{J}_{123}$} \\
\hline & & A.G.T. & A.G.L. & A.G.T. & A.G.L. & A.G.T. & A.G.L. & A.G.T. & A.G.L. & A.G.T. & A.G.L. \\
\hline \multirow{2}{*}{ A.G.C. } & $\mathrm{A}$ & 11,37 & - & 11 & 18,24 & 8,46 & 12,42 & 10,14 & 8,57 & 9,64 & 9,77 \\
\hline & B & 10,14 & - & 10,61 & 10,79 & 9,87 & 14,47 & 9,48 & 15,33 & 10,86 & 11,26 \\
\hline \multirow{2}{*}{ A.G.S. } & A & 51,46 & - & 53,14 & 58,78 & 50,94 & 60,10 & 54,22 & 51,20 & 53,57 & 51,28 \\
\hline & B & 50,92 & - & 50,06 & 40,85 & 51,60 & 53,72 & 51,24 & 50,80 & 50,60 & 51,73 \\
\hline \multirow{2}{*}{ A.G.I. } & A & 33,9 & - & 35,23 & 22,86 & 31,36 & 26,80 & 33,75 & 31,94 & 32,46 & 31,53 \\
\hline & B & 34,51 & - & 32,44 & 46,02 & 32,35 & 29,38 & 32,17 & 28,48 & 32,70 & 30,69 \\
\hline \multicolumn{6}{|c|}{$\begin{array}{l}\text { A.G.C. : acides gras à courte chaîne. } \\
\text { A.G.S. : acides gras saturés. } \\
\text { A.G.I. : acides gras insaturés. } \\
\text { A.G.T. : acides gras totaux. } \\
\text { A.G.L. : acides gras libres. }\end{array}$} & \multicolumn{6}{|c|}{$\begin{array}{l}\text { A.G.C. : short chain fatty acids. } \\
\text { A.G.S. : saturated fatty acids. } \\
\text { A.G.I. : unsaturated fatty acids. } \\
\text { A.G.T. : total fatty acids. } \\
\text { A.G.L. : free fatty acids. }\end{array}$} \\
\hline
\end{tabular}


sont les plus facilement libérés et que l'acide butyrique est préférentiellement hydrolysé.

Au cours de l'élaboration, la proportion des A.G.C. du groupe des A.G.L. de l'échantillon $\mathrm{A}$, diminue, pour atteindre, à $\mathrm{J}_{123}$, une valeur sensiblement égale à celle des A.G.C. de la fraction A.G.T. Pour l'échantillon B, l'évolution est légèrement différente; la proportion des A.G.C. de la fraction A.G.L. augmente à $J_{47}$ et $J_{77}$, puis diminue à $J_{123}$ pour atteindre les valeurs des A.G.C. de la fraction A.G.T. Cette différence peut s'expliquer par le fait que l'échantillon B subit une lipolyse plus tardive que l'échantillon A, comme l'établit l'analyse de l'indice d'acide.

Les A.G.S. représentent 50 à $51 \%$ des acides de la fraction A.G.T. du beurre et restent sensiblement à ces valeurs jusqu'à $\mathrm{J}_{123}$. Ils sont totalement absents dans la fraction A.G.L. du beurre mais apparaissent dès le $7^{\mathrm{e}}$ jour, où ils représentent $58 \%$ des acides de la fraction A.G.L. ; leur proportion atteint $60 \%$ à $\mathrm{J}_{47}$ puis diminue jusqu'à $\mathrm{J}_{123}$ pour arriver aux valeurs enregistrées pour les A.G.T. Ainsi, les A.G.S. sont moins facilement hydrolysables que les A.G.C. Au sein de la fraction des A.G.S., les acides myristique et palmitique dominent, ce qui suggère une hydrolyse préférentielle de ces acides.

Les A.G.I. représentent, environ de 33 à $35 \%$ des A.G.T. dans le beurre pour les deux échantillons. Dans la fraction A.G.L., ces acides sont absents à $\mathbf{J}_{0}$, mais apparaissent nettement dès le $7^{\mathrm{e}}$ jour, en particulier, pour l'échantillon $B$ où les A.G.I. atteignent la proportion de $46 \%$ des A.G.L., alors qu'elle n'est que de $32,4 \%$ des A.G.T. Là encore, ces acides sont plus facilement libérés. L'acide oléique est prépondérant au sein des A.G.I. De $J_{7}$ à $J_{123}$, la proportion des A.G.I. diminue pour devenir sensiblement égale à celle des A.G.I. de la fraction A.G.T.

La lipolyse observée dans le smen est un phénomène nettement sélectif. Les A.G.C. sont plus rapidement libérés, en particulier, l'acide butyrique ; viennent ensuite les A.G.I. (acide oléique) et les A.G.S. (acides myristique et palmitique). L'évolution est presque identique dans les deux lots bien que plus tardive dans l'échantillon B.

$\mathrm{Au}$ cours de la conservation, les proportions relatives de chacun des trois groupes d'acides de la fraction A.G.L. tendent à s'équilibrer avec celles de la fraction A.G.T. Ainsi, à $\mathbf{J}_{123}$, les chromatogrammes des A.G.L. s'identifient presque à ceux des A.G.T.

\section{Mesure du degré d'oxydation}

D'après le tableau 6 , l'indice de peroxyde qui est de $0,26 \mathrm{meq} / \mathrm{kg}$ de matière grasse à $\mathrm{J}_{0}$, s'élève à 1,43 à $\mathrm{J}_{123}$ pour I'échantillon $\mathrm{A}$; il est de 0,20 à $\mathrm{J}_{0}$ et atteint la valeur de 1,12 à $\mathrm{J}_{123}$, pour l'échantillon $\mathrm{B}$.

Les teneurs en carbonylés totaux saturés sont, pour l'échantillon A, de 6,899 millimole $/ \mathrm{kg}$ de matière grasse à $\mathrm{J}_{0}$ et 8,026 à $\mathrm{J}_{123}$, pour l'échantillon $\mathrm{B}$, de 5,628 à $J_{0}$ et 7,050 à $J_{123}$. Les teneurs en carbonylés totaux insaturés sont inférieures : elles sont, pour l'échantillon $\mathrm{A}$, de 1,63 à $\mathrm{J}_{0}$ et 3,21 à $\mathrm{J}_{123}$ pour l'échantillon $\mathrm{B}$, de 1,365 à $\mathrm{J}_{0}$ et 2,7 à $\mathrm{J}_{123}$. 
TABleau 6 - TABLe 6

Etude du degré d'oxydation du smen - Study of the oxidation level of smen

\begin{tabular}{|c|c|c|c|c|}
\hline \multirow{2}{*}{$\begin{array}{l}\text { Prélève- } \\
\text { ment }\end{array}$} & \multirow{2}{*}{$\begin{array}{l}\text { Echan- } \\
\text { tillon }\end{array}$} & \multirow{2}{*}{$\begin{array}{c}\text { Indice } \\
\text { de peroxyde } \\
\text { meq/kg MG }\end{array}$} & \multicolumn{2}{|c|}{ Carbonylés totaux } \\
\hline & & & $\begin{array}{c}\text { saturés } \\
\text { millimole/kg MG }\end{array}$ & $\begin{array}{c}\text { insaturés } \\
\text { millimole/kg MG }\end{array}$ \\
\hline $\begin{array}{l}\text { Beurre } \\
0 \mathrm{j}\end{array}$ & $\begin{array}{l}\mathrm{A} \\
\mathrm{B}\end{array}$ & $\begin{array}{l}0,26 \\
0,20\end{array}$ & $\begin{array}{l}6,899 \\
5,628\end{array}$ & $\begin{array}{l}1,163 \\
1,365\end{array}$ \\
\hline $\begin{array}{l}\text { Smen } \\
7 \mathbf{j}\end{array}$ & $\begin{array}{l}\mathrm{A} \\
\mathrm{B}\end{array}$ & $\begin{array}{l}0,29 \\
0,26\end{array}$ & $\begin{array}{l}6,643 \\
6,274\end{array}$ & $\begin{array}{l}1,494 \\
1,541\end{array}$ \\
\hline $\begin{array}{l}\text { Smen } \\
47 \mathrm{j}\end{array}$ & $\begin{array}{l}\mathrm{A} \\
\mathrm{B}\end{array}$ & $\begin{array}{l}0,50 \\
0,44\end{array}$ & $\begin{array}{l}9,318 \\
8,274\end{array}$ & $\begin{array}{l}2,425 \\
2,388\end{array}$ \\
\hline $\begin{array}{l}\text { Smen } \\
77 \mathrm{j}\end{array}$ & $\begin{array}{l}\mathrm{A} \\
\mathrm{B}\end{array}$ & $\begin{array}{l}1,29 \\
0,99\end{array}$ & $\begin{array}{l}8,092 \\
7,720\end{array}$ & $\begin{array}{l}3,041 \\
2,840\end{array}$ \\
\hline $\begin{array}{l}\text { Smen } \\
123 \mathrm{j}\end{array}$ & $\begin{array}{l}\mathrm{A} \\
\mathrm{B}\end{array}$ & $\begin{array}{l}1,43 \\
1,12\end{array}$ & $\begin{array}{l}8,026 \\
7,050\end{array}$ & $\begin{array}{l}3,210 \\
2,700\end{array}$ \\
\hline
\end{tabular}

TABleaU 7 - TABle 7

Etude des teneurs en diacétyle et acétoïne du smen (en p.p.m.*)

Diacetyl and acetoin composition of smen

\begin{tabular}{|c|c|c|c|}
\hline Prélèvement & Echantillon & Diacétyle & Diacétyle + Acétoïne \\
\hline $\begin{array}{l}\text { Beurre } \\
0 \mathrm{j}\end{array}$ & $\begin{array}{l}\mathrm{A} \\
\mathrm{B}\end{array}$ & $\begin{array}{l}3,20 \\
3,15\end{array}$ & $\begin{array}{l}6,00 \\
5,90\end{array}$ \\
\hline $\begin{array}{l}\text { Smen } \\
7 \mathrm{j}\end{array}$ & $\begin{array}{l}\mathrm{A} \\
\mathrm{B}\end{array}$ & $\begin{array}{l}2,90 \\
1,56\end{array}$ & $\begin{array}{l}4,20 \\
4,02\end{array}$ \\
\hline $\begin{array}{l}\text { Smen } \\
47 \mathrm{j}\end{array}$ & $\begin{array}{l}\mathrm{A} \\
\mathrm{B}\end{array}$ & $\begin{array}{l}\text { traces } \\
\text { traces }\end{array}$ & $\begin{array}{l}0,18 \\
0,16\end{array}$ \\
\hline $\begin{array}{l}\text { Smen } \\
77 \mathrm{j}\end{array}$ & $\begin{array}{l}\mathrm{A} \\
\mathrm{B}\end{array}$ & $\begin{array}{l}\text { traces } \\
\text { traces }\end{array}$ & $\begin{array}{l}0,41 \\
0,12\end{array}$ \\
\hline $\begin{array}{l}\text { Smen } \\
123 \mathrm{j}\end{array}$ & $\begin{array}{l}\mathrm{A} \\
\mathrm{B}\end{array}$ & $\begin{array}{l}\text { traces } \\
\text { traces }\end{array}$ & $\begin{array}{l}\text { traces } \\
\text { traces }\end{array}$ \\
\hline
\end{tabular}

p.p.m.* : Partie par million. - Part per million. 
Ces valeurs montrent que le smen est un produit peu oxydé. En comparant les deux échantillons, nous remarquons, cependant, que l'échantillon A est plus oxydé que $\mathrm{B}$, ce qui, dans le cas particulier, est très relatif.

\section{Dosage du diacétyle et de l'acétoïne}

Les teneurs en diacétyle sont, respectivement, pour les échantillons A et $\mathrm{B}$ de 3,2 p.p.m. et 3,15 p.p.m. à $\mathrm{J}_{0}$ (tab. 7). A partir de $\mathrm{J}_{47}$, le diacétyle n'existe plus qu'à l'état de traces. L'acétoïne est également transformé mais sa disparition n'est remarquée qu'à $\mathrm{J}_{123}$. A $\mathbf{J}_{0}$, les teneurs en diacétyle + acétoïne sont de 6 p.p.m pour B ; elles diminuent au cours de la conservation pour atteindre à $\mathrm{J}_{77}$ des valeurs de 0,41 p.p.m. pour $\mathrm{A}$ et 0,12 p.p.m. pour B.

\section{Discussions}

\section{A. Les modifications chimiques de la phase grasse}

\section{La lipolyse}

L'analyse du smen provenant des différents points de vente ainsi que l'étude du produit au cours de son élaboration, montrent que la transformation importante de la phase grasse se réalise par la lipolyse.

Dans le produit fini, l'intensité de l'hydrolyse de la matière grasse, exprimée en indice d'acide, est variable d'un échantillon à l'autre. Cette variation peut être attribuée à l'âge différent des échantillons. Cependant, en examinant les échantillons, individuellement, du point de vue de la composition en acides gras libres, il apparaît que l'hydrolyse est, du point de vue qualitatif, pratiquement identique pour tous les échantillons et sélective.

En effet, au cours de l'évolution de la matière grasse en fonction de la durée de conservation, les acides gras à courte chaîne (A.G.C.) de $\mathrm{C}_{4} \mathrm{à}_{10}$ sont plus facilement libérés et l'acide butyrique est préférentiellement hydrolysée. RICHARDS et El-SADEK (1949), en étudiant le rôle des micro-organismes dans le rancissement du beurre, observent, également, que les A.G.C. sont plus rapidement libérés et que les bactéries produisent une plus grande proportion d'acides butyrique et caproïque que les moisissures. Après les A.G.C., ce sont les acides gras insaturés (A.G.I.) de $\mathrm{C}_{14}$ à $\mathrm{C}_{18}$ qui sont préférentiellement libérés. Au sein de cette fraction, l'acide oléique est prépondérant. Les acides gras saturés (A.G.S.) de $\mathrm{C}_{12}$ à $\mathrm{C}_{20}$ sont libérés en dernier. Au sein de cette fraction, les acides myristique et palmitique dominent, ce qui suggère une hydrolyse préférentielle de ces acides.

Les résultats obtenus concernant la cinétique de l'attaque de la matière grasse sont conformes aux études de spécificité de la lipase du lait et de la majorité des lipases microbiennes (BROCKEROFF et al., 1974). D'après ces études, les A.G.C. et les A.G.I. sont plus facilement hydrolysés.

L'acidité des différents échantillons augmente avec la durée de conservation. Cette dernière est donc un facteur important dans la fabrication du 
smen. Cependant, la vitesse de décomposition diminue avec le temps de conservation. En effet, au cours de l'élaboration du produit, les proportions relatives de chacun des trois groupes d'acides (A.G.C., A.G.I. et A.G.S.) de la fraction acides gras libes (A.G.L.) tendent à s'équilibrer avec celles de la fraction des acides gras totaux (A.G.T.) (tab. 5). Ainsi, à la fin de la période de formation, les chromatogrammes des A.G.L. s'identifient presque à ceux des A.G.T. Ce ralentissement s'explique par l'effet inhibiteur des A.G.L. sur la production des lipases par les micro-organiques lipolytiques (SMith et Alford, 1966).

Au cours de l'élaboration, la proportion des acides gras à courte chaîne de la fraction A.G.T. baisse, alors qu'elle devrait rester sensiblement constante. Les A.G.T. comprennent, en effet, aussi bien les acides gras estérifiés que ceux existant à l'état libre. Cette diminution est beaucoup plus nette pour l'acide butyrique, qui est plus hydrosoluble que les autres acides gras à courte chaîne, et doit correspondre, au moins en partie, à la perte de ces acides dans l'eau exsudée. Peut-être aussi, faut-il faire intervenir l'hydro-solubilité de ces acides, en particulier, les acides butyrique et caproïque, qui rend leur extraction difficile au moment de leur dosage (SALIH et al., 1977).

La lipolyse du smen est précoce puisqu'au $7^{\mathrm{e}}$ jour après salage, les indices d'acide sont déjà relativement élevés. Dans cette action, les lipases microbiennes jouent le rôle essentiel car la lipase naturelle du lait est sensible au salage (DEeTH et Fitz-Gerald, 1976).

Malgré l'addition de sel, les processus d'hydrolyse ne sont pas arrêtés. La teneur moyenne en sel est de $1,5 \%$ correspondant à une concentration dans la phase aqueuse de $8 \%$, teneur qui permet encore à certains microorganismes de croître. Même un salage à $10 \%$ suivi d'un entreposage à $+5^{\circ} \mathrm{C}$ n'empêchent pas 1'hydrolyse du beurre (SHéRIF et al., 1977).

\section{L'oxydation}

Malgré le salage et la lipolyse deux facteurs qui favorisent l'oxydation (Mac Dowell, 1955 ; Sambuc et al., 1971 ; Deeth et Fitz-Gerald, 1976), le smen reste jusqu'au bout peu oxydé. A $\mathrm{J}_{123}$, l'indice de peroxyde (I.P.) est le plus élevé, il est de $1,43 \mathrm{meq} / \mathrm{kg}$ de matière grasse pour l'échantillon $\mathrm{A}$ et 1,12 pour B (tab. 6). Or, en général, un produit est considéré comme oxydé lorsque son I.P. est supérieur à 3 meq par kg de matière grasse. Sur 19 échantillons du smen de commerce, 14 soit $73 \%$, ont un indice de peroxyde inférieur à 3 .

Cette faible oxydation s'explique par les conditions de conservation du produit : entreposage à l'abri de l'air et de la lumière. Une forte oxydation n'a pu être observée que sur quelques échantillons du commerce. On peut légitimement la supposer due à un contact prolongé avec l'oxygène de l'air au moment de la vente. Cette augmentation est plus importante pour les échantillons ayant un indice d'acide ou une teneur en sel élevée. $C^{\prime}$ est ainsi que l'échantillon A dont l'indice d'acide est plus élevé que B, apparaît relativement plus oxydé. 


\section{B. Les modifications chimiques de la phase aqueuse}

L'analyse de la composition de la phase aqueuse du smen montre une diminution générale des teneurs des divers constituants au cours de la conservation. L'exsudation de la phase aqueuse entraîne une augmentation proportionnelle des teneurs en matière grasse et en extrait sec délipidé.

Les constituants organiques, de la phase aqueuse (lactose et protéines) subissent une chute importante (tab. 3). Celle-ci est la conséquence d'une dégradation microbienne, mais une partie du lactose et des protéines est perdue avec de l'eau exsudée. En effet, au cours de la conservation du produit, une partie de la fraction aqueuse exsude et vient sourdre à la surface du smen. Cette fraction est, généralement, éliminée au moment de la vente du smen. Avec l'eau, les éléments dissous de la phase aqueuse sont également éliminés.

La même évolution s'applique au sel, soit une diminution de $J_{0}$ à $J_{123}$ avec, cependant, une exception à $\mathrm{J}_{7}$, une augmentation due à l'opération de salage.

Le taux de diacétyle (tab. 7), substance élaborée par certains streptocoques lactiques à partir du lactose et des citrates et considérée comme l'un des principaux responsables de l'arôme du beurre, diminue au cours de l'élaboration.

Dans le beurre fermier marocain, les teneurs en diacétyle sont de l'ordre de 3 p.p.m., comparées à celles du beurre industriel (1,5-2 p.p.m.), elles sont élevées. Au cours de l'élaboration du smen, il se produit une diminution, voire une disparition de cette substance, due à la destruction du diacétyle déjà formé. En effet, PACK et al. (1968) ont montré qu'aux températures comprises entre 15 et $20^{\circ} \mathrm{C}$, qui sont, généralement, celles de la conservation du smen, la destruction du diacétyle est maximale. Les conditions d'anaérobiose au cours de la conservation du smen rendent, probablement, le milieu réducteur, ce qui favorise la transformation du diacétyle en butanédiol.

La disparition, dès le $47^{e}$ jour de conservation, indique que cette substance n'est pas responsable de l'arôme du smen.

\section{Références bibliographiques}

Brockerhoff H., Jensen R.G., 1974. Lipolytic enzyme. Academic Press, New York.

Deeth H.C., Fitz-Gerald C.H., 1976. Lipolysis in dairy products. A review. Aust. J. Dairy Technol., 31, 53-64.

Kuzdzal-Savoie S., Kuzdzal W., Langlots D., 1971. Dosage des acides gras libres. Lait, 51, 534-544.

Mac DoweIL A.K.R., 1955. The effects of salts and of anti-oxydants on the keeping quality of butter. J. Dairy Res., 22, 349-364.

Pack M.Y., Vedamuthm E.R., Sandine W.E., Elliker P.R., Leesment H., 1968. Effect of temperature on growth and diacetyl production by aroma production in a single and mixed-strain lactic cultures. J. Dairy Sci., 51, 339-344. 
Prill E.A., Hammer B.W., 1938. A colorimetric method for determination of diacetyl. Iowa State college. J. Sci., 12, 385.

Richards T., El-SadeK G.M., 1949. The nature and quality of fatty acids produced in butter fat by the action of micro-organisms. J. Dairy Res., 16, 46-52.

Salih A.M.A., Anderson G., TuckLey B., 1977. The determination of short-and long-chain free fatty acids in milk. J. Dairy Res., 44, 601-605.

Sambuc E., Reymond G., Nauder M., 1971. A propos de l'oxydation des margarines salées. Rev. Fr. Corps Gras, 18, 17-20.

Sherif R.M., Ibrahim S.S., El-Ghandour M.A., AbDelhamid L.B., Younes M., 1977. Detection of deterioration in some dairy products. Ann. Agric. Sci., 8, 91-98.

SMith J.L., Alford J.A., 1966. Inhibition of microbial lipases by fatty acids. Appl. Microbiol., 14, 699-705.

Wolf J.P., 1968. Manuel d'analyse des corps gras. Ed. Azoulay, Paris. 